\title{
Diagnostic and prognostic utility of molecular markers in synchronous bilateral breast carcinoma
}

\author{
Reda S Saad ${ }^{1}$, Krista L Denning ${ }^{1}$, Sydney D Finkelstein ${ }^{2}$, Yulin Liu ${ }^{1}$, Telma C Pereira ${ }^{1}$, \\ Xiaoqi $\operatorname{Lin}^{1}$ and Jan F Silverman ${ }^{1}$ \\ ${ }^{1}$ Department of Pathology, Allegheny General Hospital/Drexel University College of Medicine, Pittsburgh, PA, \\ USA and ${ }^{2}$ RedPath Inc., Pittsburgh, PA, USA
}

\begin{abstract}
Histologic criteria have a limited role in determining whether the synchronous bilateral breast carcinomas represent two primaries or a metastasis to the contralateral breast. We studied the molecular analysis of synchronous bilateral breast carcinoma and whether they are originating from a single or different clone. We examined 17 patients with breast carcinoma, including 12 patients with synchronous bilateral carcinomas and control group of 5 infiltrating ductal carcinomas with regional lymph node metastases. Mutations were quantitatively determined to detect loss of heterozygosity (LOH) and microsatellite size alterations for a broad panel of 15 markers, involving 10 chromosomes using polymerase chain reaction. The carcinomas were classified as de novo or metastasis based on three levels of concordance: (1) marker-affected tumors were considered concordant if $50 \%$ or more of the same markers were mutated, (2) same gene copy affected, and (3) temporal sequence of mutation acquisition. In synchronous bilateral breast carcinoma patients, molecular analysis showed discordant mutations in all cases, supporting the diagnosis of de novo bilateral primary breast carcinomas. In patients with lymph node metastases, the primary breast carcinoma and metastases shared the same mutations, revealing a metastatic lesion. In conclusion, the application of molecular technology may play an important role for the differential diagnosis of dual primary carcinomas vs a metastatic breast cancer to contralateral breast. In this study, synchronous bilateral breast cancers represent two independent primaries rather than metastatic events.
\end{abstract}

Modern Pathology (2008) 21, 1200-1207; doi:10.1038/modpathol.2008.35; published online 9 May 2008

Keywords: synchronous bilateral breast carcinoma; molecular pathology; LOH

Contralateral breast carcinoma is the most common second malignancy for breast carcinoma patients, with an incidence as high as $12 \% \cdot{ }^{1-3}$ Bilateral breast carcinomas exist in two forms, synchronous, in which both tumors occur at the same time, or metachronous, in which the carcinomas occur at different times. Synchronous bilateral breast carcinoma is uncommon, reported to range from 0.3 to $8 \%$ in the literature. ${ }^{4-7}$ This wide range of reported incidence rates for synchronous bilateral breast carcinoma is a result of different time cutoff. Although some authors require both breast carcinomas to be diagnosed simultaneously or within less

Correspondence: Dr RS Saad, MD, PhD, Department of Pathology and Laboratory Medicine, Allegheny General Hospital, 3rd floor, south tower, 320 East North Avenue, Pittsburgh, PA 15212, USA. E-mail: rsaad@wpahs.org

Received 02 February 2007; revised 31 May 2007; accepted 05 June 2007; published online 9 May 2008 than 1 month, ${ }^{7-10}$ others used up to 12 months as a cutoff time to differentiate synchronous from metachronous bilateral breast carcinomas. $^{11-13}$ Using criteria of less than 1 month, the incidence of synchronous bilateral breast is less than $2 \% .^{7,14}$

When cancer is detected in the opposite breast, the question arises whether this tumor is a second cancer (polyclonal origin) or a metastatic spread from the first breast cancer (monoclonal origin). The answer to this question is critical as both tumor staging and management will be different depending upon the results. ${ }^{11}$ Currently, this distinction is attempted using histopathologic features. Different histologic types, a better histologic differentiation, presence of in situ component, and absence of metastatic spread would favor a second primary. ${ }^{8,15,16}$ In addition, long time interval between the tumor onsets may be regarded as a proof of the true bilaterality. However, in breast cancer patients, there are many exceptions where the latency 
intervals for metastases or recurrence might be as long as 20 years. ${ }^{15}$

Accumulating literature presents contradicting results. ${ }^{11,17-25}$ Some authors have demonstrated similarity in histologic subtype, ${ }^{12,23}$ tumor grade, ${ }^{17}$ and hormone receptor status ${ }^{16,18}$ between the two tumors, suggesting a single-cell origin, whereas others had different results. ${ }^{25}$ In addition, similar mammographic appearance may be seen in bilateral breast carcinomas with a mirror image location. ${ }^{26,27}$ Neither clinical nor histopathologic features can definitively determine the relationship between multiple deposits of breast cancer at the individual patient level with certainty. Using molecular techniques, few studies showed the presence of similarities in bilateral breast cancer, indicating that these carcinomas may result from a metastatic event, ${ }^{11,24}$ while other investigators present evidence for the independent pathogenesis in majority of these tumors. ${ }^{16,19-22,25}$ This controversy reflects the lack of a definitive methodology to distinguish de novo primary from metastatic tumor deposition. Comparative mutational profiling offers a novel and highly detailed means to understand the relationship between different tumors deposits suitable for clinical application in surgical pathology. This technique has the potential to differentiate contralateral breast metastases from a second primary, with prognostic prediction.

In this study, we chose to employ the loss of heterozygosity (LOH) studies to examine for microsatellite alterations in each of the synchronous bilateral breast carcinoma, using a broad panel of mutational markers with quantitative molecular analysis. This approach can also provide valuable information on the molecular pathogenesis of breast cancer.

\section{Materials and methods}

Computer search identified 12 patients with synchronous bilateral breast carcinoma, defined as when both tumors were diagnosed either simultaneously or within a time period of 1 month (between January 1997 to December 2001). Twenty-four archival routinely processed, formalin-fixed, and paraffin-embedded surgical pathology specimens from lumpectomy/mastectomy were retrieved from the pathology files of the Allegheny General Hospital. We also included five breast cancer patients with axillary lymph nodes metastases as a control group. Institutional Board review approved the study.

The hematoxylin and eosin-stained slides were evaluated for histologic type, grade, presence of lymphovascular invasion, in situ component, and peritumoral lymphoplasmacytic reaction, without knowledge of patient information or molecular analysis results. A histologic comparison between the bilateral tumors of each patient was also performed to assess for any cytologic or architectural similarities or differences.

From each case, a paraffin block that included the breast carcinoma and normal adjacent breast tissue was selected for genotypic analysis. Slides were sectioned from the block (one hematoxylin and eosin section and six unstained deparaffinized sections followed by another hematoxylin and eosin section). The hematoxylin and eosin slides were examined, and tumor targets were selected and marked for microdissection under direct visualization, using a stereoscopic microscope and a beveled surgical blade. One to seven targets were chosen within each lesion to include different histologic growth patterns and to assess for intralesional heterogeneity.

Allelic imbalance for a broad panel of microsatellite markers in proximity to known tumor suppressor genes was quantitated using automated PCR/gel electrophoresis. A total of 15 specific microsatellite regions from 10 chromosomal sites, including 1p, 3p, 5q, 9p, 9q, 10q, 17p, 17q, 19q, and $22 q$, were evaluated. Normal microdissected tissue samples were first evaluated to determine whether the patient's DNA was informative at each specific marker locus. When a particular microsatellite marker in a normal tissue sample manifested only a single peak, the patient was designated as noninformative for that marker. For informative subjects, with respect to a specific marker, alleles were assessed as being in balance when the ratio of the individual alleles peaks fell within two standard deviations of the average generated from a large series of non-neoplastic microdissected tissue samples for subjects with the same pairing of specific polymorphic alleles.

In all cases, microdissected normal tissue samples were taken to be no-larger in size and contain no more cells than the smallest microdissected lesional sample. Fluorescent-labeled oligonucleotide primers were employed for quantitative determination of allelic imbalance based on the peak height ratio of polymorphic microsatellite alleles. Post-amplification products were electrophoresed and relative fluorescence determined for individual allele peak height (GeneScan, ABI3100, Applied Biosystems, Foster City, CA, USA). The ratio of peaks was calculated by dividing the value for the shorter-sized allele by that of the longersized allele (Figure 1). The genetic loci selected for PCR included short tandem repeats that are known to be at or near specific tumor suppressor genes. The short tandem repeats were selected for their high rates of polymorphism (ideally $>75 \%$ ) in the general population. PCR reactions and subsequent analysis were repeated and confirmed in all cases, to avoid misinterpreting allelic dropout, such as LOH. Thus, it was possible to assign a status as being either non-informative, in allelic balance (no $\mathrm{LOH}$ ) or positive for imbalance $(\mathrm{LOH})$, with the latter further characterized as being 


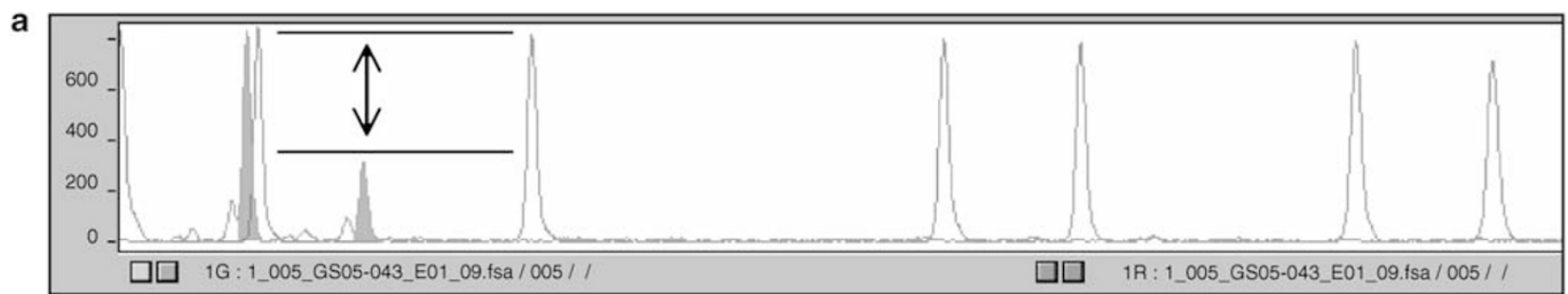

b

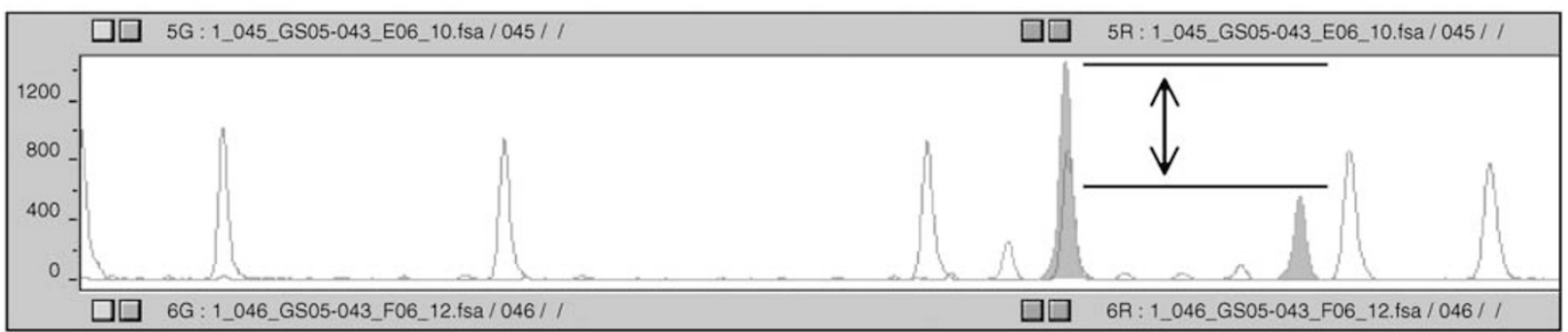

Figure 1 Allelic imbalance analysis of microdissected breast tumor. Both images show imbalance of one allele, indicating mutation. (The ratio of peak heights is two s.d. beyond the range determined by analyzing a large number of non-neoplastic tissue samples from patients with identical allele pairing) (Histogram).

in relative deficiency of the shorter (red) or longer (blue) allele.

The temporal sequence of mutation acquisition can be determined by the percentage of mutated cells at a given site. This in turn was based upon a simple model of clonal expansion with forward progression in malignant phenotype of tumor cells. Mutations acquired earlier in time were more likely to present across a wide distance of tumor encompassing all microdissection targets for a given tumor deposit. At the same time, mutations acquired earlier in time were more likely to be clonally expanded and this was present in a higher percentage of cells than mutations acquired later.

For each microdissected focus, the fractional allelic loss (FAL) was calculated by dividing the number of markers with $\mathrm{LOH}$ by the total number of informative markers. For each case, mean and maximum FALs were recorded. For each marker, allelic imbalance rate in each diagnostic category was calculated by dividing the number of cases with $\mathrm{LOH}$ in any microdissected focus by the total number of cases informative for that marker.

\section{Results}

Table 1 summarizes the clinicopathologic findings in our patients included in this study. The average age of the patients was $69.5 \pm 10.9$ years (range, 49 81, with a median 74 years). Ten patients had bilateral mastectomy; either simple (8 cases) or modified radical mastectomy (3 cases), while the remaining two patients had bilateral lumpectomy. Sentinel lymph nodes were performed in all cases. Tumor size ranged between 0.3 to $4.0 \mathrm{~cm}$, with a mean value of $1.6 \mathrm{~cm}$ and median $1.5 \mathrm{~cm}$. Two cases were lymph node positive, one case with one positive lymph node and the second case with 2 positive lymph nodes.

Five invasive carcinomas were graded 1, 11 cases graded 2, and 8 cases graded 3 . Five cases showed different histologic features between the two tumors suggesting two independent primaries. Two cases showed ductal carcinoma with different histologic types of both tumors (NOS type vs mucinous and tubular differentiation). The other three cases showed invasive lobular carcinoma (two classic, nuclear grade 1 and one pleomorphic lobular with intermediate nuclear grade) on one side, and invasive ductal carcinoma on the other side of breast. In situ component was present in both synchronous bilateral breast carcinoma in five cases, and identified in only one of the tumors, in the other three cases. A host reaction was identified in three cases. A synchronous bilateral breast carcinoma, with similar histologic type was present in $7 / 12$ $(58 \%)$ cases, suggesting the possibility of metastasis (Figure 2).

Estrogen receptors (ER) were positive in 21/24 $(87.5 \%)$, progesterone receptors (PgR) were positive in $17 / 24(71 \%)$, and Her2-neu amplification in 6/24 $(25 \%)$ patients. ER, PgR and Her2-neu showed similar results in both synchronous bilateral breast carcinomas in $8 / 12(67 \%)$ patients, while different results were present in the remaining 4/12 (33\%) patients.

Detailed allelic imbalance information was obtained from each microdissected tissue target, producing a detailed fingerprint of mutational damage in each case. Figure 3 details the acquired $\mathrm{LOH}$ mutations in cumulative amount, specific alleles affected by imbalance (red vs blue) and in percentage of affected tumor cells for a given mutational marker. No single marker or genomic site used in this study avoided mutational change in 
Table 1 Clinicopathologic results of our 12 synchronous bilateral breast cancer cases

\begin{tabular}{|c|c|c|c|c|c|c|c|c|}
\hline No. & Age & Duration & Histologic & Grade & In situ & $L N$ & Procedure & IHC markers \\
\hline \multirow[t]{2}{*}{1} & 59 & 0 & IDC,NOS & $3 / 3$ & Absent & Negative & Bilateral & ER+ve,PR-ve,Her2+ve \\
\hline & & & IDC,NOS & $3 / 3$ & Absent & Negative & Mastectomy & ER-ve,PR+ve,Her2+ve \\
\hline \multirow[t]{2}{*}{2} & 81 & 0 & IDC,NOS & $2 / 3$ & Present & Negative & Bilateral & $\mathrm{ER}+\mathrm{ve}, \mathrm{PR}+\mathrm{ve}, \mathrm{Her} 2$-ve \\
\hline & & & IDC,NOS & $2 / 3$ & Present & Negative & Mastectomy & ER+ve,PR+ve,Her2-ve \\
\hline \multirow[t]{2}{*}{3} & 49 & 0 & IDC,NOS & $3 / 3$ & Present & Positive $^{2}$ & Bilateral & ER+ve,PR+ve,Her2+ve \\
\hline & & & IDC,NOS & $2 / 3$ & Present & Negative & Mastectomy & $\mathrm{ER}+\mathrm{ve}, \mathrm{PR}+\mathrm{ve}, \mathrm{Her} 2$-ve \\
\hline \multirow[t]{2}{*}{4} & 74 & 0 & ILC,Classic & $1 / 3$ & Present & Negative & Bilateral & $\mathrm{ER}+\mathrm{ve}, \mathrm{PR}+\mathrm{ve}, \mathrm{Her} 2$-ve \\
\hline & & & IDC,NOS & $2 / 3$ & Present & Positive $^{1}$ & Mastectomy & ER+ve,PR+ve,Her2-ve \\
\hline \multirow[t]{2}{*}{5} & 78 & 0 & IDC,Tubular & $2 / 3$ & Absent & Negative & Bilateral & ER+ve,PR+ve,Her2-ve \\
\hline & & & IDC,NOS & $2 / 3$ & Absent & Negative & Lumpectomy & ER+ve,PR+ve,Her2-ve \\
\hline \multirow[t]{2}{*}{6} & 80 & 0 & IDC,NOS & $3 / 3$ & Present & Negative & Bilateral & ER-ve,PR-ve,Her2+ve \\
\hline & & & ILC,Pleom. & $2 / 3$ & Absent & Negative & Mastectomy & $\mathrm{ER}+\mathrm{ve}, \mathrm{PR}+\mathrm{ve}, \mathrm{Her} 2$-ve \\
\hline \multirow[t]{2}{*}{7} & 63 & 0 & IDC,NOS & $2 / 3$ & Absent & Negative & Bilateral & $\mathrm{ER}+\mathrm{ve}, \mathrm{PR}+\mathrm{ve}, \mathrm{Her} 2$-ve \\
\hline & & & IDCa,Mucinous & $2 / 3$ & Present & Negative & Mastectomy & $\mathrm{ER}+\mathrm{ve}, \mathrm{PR}+\mathrm{ve}, \mathrm{Her} 2$-ve \\
\hline \multirow[t]{2}{*}{8} & 66 & 0 & IDCa,NOS & $3 / 3$ & Present & Negative & Bilateral & ER+ve,PR-ve,Her2+ve \\
\hline & & & ILCa,Classic & $1 / 3$ & Present & Negative & Mastectomy & ER+ve,PR-ve,Her2-ve \\
\hline \multirow[t]{2}{*}{9} & 75 & 10 days & IDCa,NOS & $3 / 3$ & Absent & Negative & Bilateral & ER-ve,PR-ve,Her2+ve \\
\hline & & & IDCa,NOS & $2 / 3$ & Present & Negative & Lumpectomy & $\mathrm{ER}+\mathrm{ve}, \mathrm{PR}+\mathrm{ve}, \mathrm{Her} 2$-ve \\
\hline \multirow[t]{2}{*}{10} & 74 & 0 & IDCa,NOS & $1 / 3$ & Absent & Negative & Bilateral & ER+ve,PR-ve,Her2-ve \\
\hline & & & IDCa,NOS & $1 / 3$ & Absent & Negative & Mastectomy & ER+ve,PR-ve,Her2-ve \\
\hline \multirow[t]{2}{*}{11} & 54 & 0 & IDCa,NOS & $2 / 3$ & Present & Negative & Bilateral & ER+ve,PR+ve,Her2-ve \\
\hline & & & IDCa,NOS & $3 / 3$ & Present & Negative & Mastectomy & $\mathrm{ER}+\mathrm{ve}, \mathrm{PR}+\mathrm{ve}, \mathrm{Her} 2$-ve \\
\hline \multirow[t]{2}{*}{12} & 57 & 0 & IDCa,NOS & $2 / 3$ & Absent & Negative & Bilateral & $\mathrm{ER}+\mathrm{ve}, \mathrm{PR}+\mathrm{ve}, \mathrm{Her} 2$-ve \\
\hline & & & IDCa,NOS & $2 / 3$ & Absent & Negative & Mastectomy & ER+ve,PR+ve,Her2-ve \\
\hline
\end{tabular}

$\mathrm{ER}=$ estrogen receptor; Her2 $=$ Her2neu receptor; $\mathrm{IDCa}=$ invasive ductal carcinoma; $\mathrm{IHC}=$ immunohistochemical markers; ILCa $=$ invasive lobular carcinoma; $\mathrm{LN}=$ lymph node; $\mathrm{NOS}=$ not otherwise specified; $\mathrm{PR}=$ progesterone receptor.
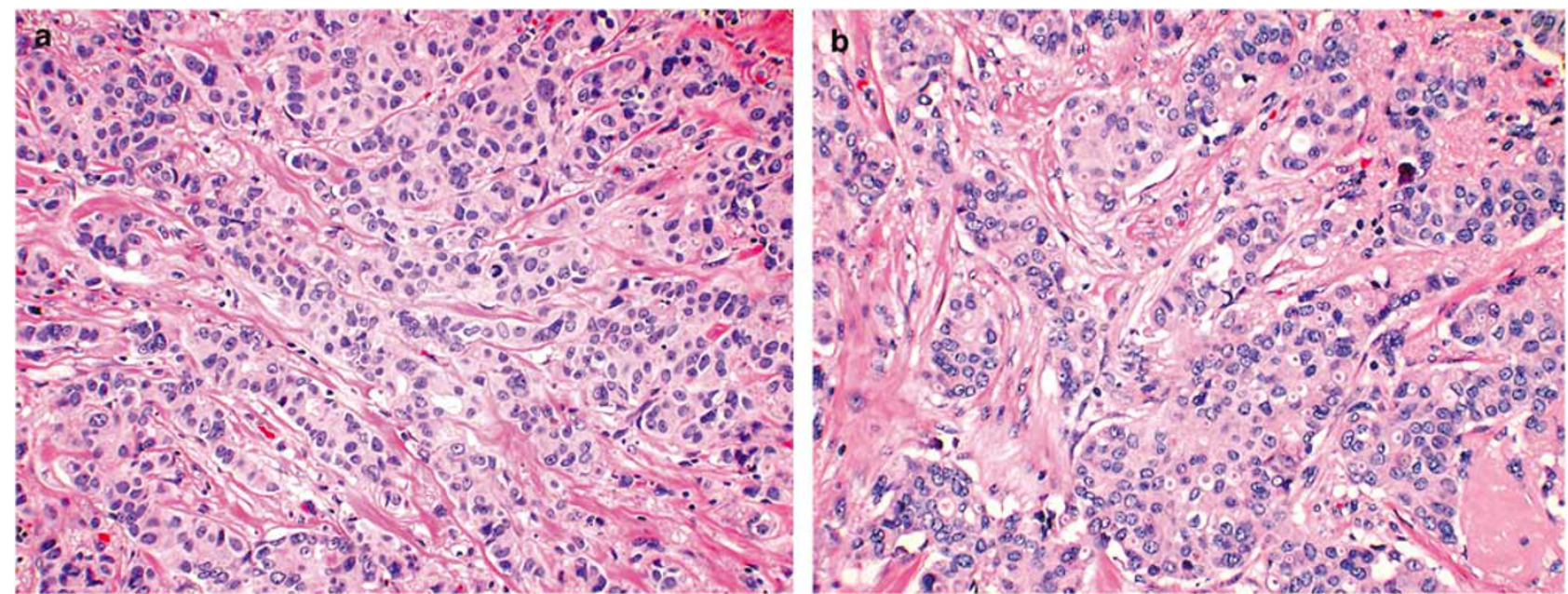

Figure 2 Histology of both tumors in case number 11. Notice the histologic similarity between both tumors. Both tumors also show similar ER, PR and Her2neu results. Molecular studies proved that these two lesions are independent, and not metastatic (Hematoxylin \& Eosin $\times 400$ ).

at least two patients. While certain markers were found to be more frequently involved, that is, 9p21 and $17 q 21$ compared to others (ie $5 q 23,17 p 13$ ), no single pattern of mutation acquisition or involvement was evident. These findings suggest that breast cancer develops using various pathways that have unique and personalized patterns of mutational change.

On the basis of the distinction between de novo primary cancer formation vs metastatic tumor deposition on a mutational profile, it was clear that this discrimination could be readily arrived in all cases (Figure 3). Shared mutations that were present in a high percentage of affected tumor cells included the affected specific allele copy, indicate single cancers with metastatic spread (patients 1, 2, 4, and 5) (Figure 3). Conversely, cancers that manifested a discordant profile, especially for early acquired mutations, were independent primary cancers. Particularly noteworthy in this regard are patients 6,15 , and 16 , where unique markers were mutated in separate tumors but the specific allele copies affected by imbalance were different. This provided a very strong support for independent primary 


\begin{tabular}{|c|c|c|c|c|c|c|c|c|c|c|c|c|c|c|c|c|c|c|c|}
\hline \multirow{2}{*}{ Patient } & \multirow{2}{*}{ Loc } & 1 & 2 & 3 & 4 & 5 & 6 & 7 & 8 & 9 & 10 & 11 & 12 & 13 & 14 & 15 & \multirow{2}{*}{$\begin{array}{l}\text { Positive } \\
\text { Markers }\end{array}$} & \multirow{2}{*}{$\begin{array}{c}\text { Informative } \\
\text { Markers }\end{array}$} & \multirow{2}{*}{$\begin{array}{l}\text { Shared } \\
\text { Markers }\end{array}$} \\
\hline & & $1 \mathrm{p} 36$ & 1p36 & $3 \mathrm{p} 26$ & $3 \mathrm{p} 26$ & $5 q 23$ & $5 q 23$ & $9 \mathrm{p} 21$ & \begin{tabular}{|l|}
$9 p 21$ \\
\end{tabular} & $10 \mathrm{q} 23$ & $10 \mathrm{q} 23$ & $17 \mathrm{p} 13$ & $17 \mathrm{p} 13$ & $17 q 21$ & 21922 & 22q13 & & & \\
\hline \multirow{2}{*}{1} & B & NO LOH & NO LOH & $\mathrm{NI}$ & $73 \%$ & NO LOH & NO LOH & NO LOH & NO LOH & $91 \%$ & $\mathrm{NI}$ & NO LOH & NO LOH & NO LOH & NOLOH & NO LOH & 2 & 13 & \multirow{2}{*}{1} \\
\hline & $M$ & NO LOH & NO LOH & $\mathrm{NI}$ & NO LOH & NO LOH & NO LOH & NO LOH & NO LOH & $57 \%$ & $\mathrm{NI}$ & NO LOH & NO LOH & NO LOH & NOLOH & NO LOH & 1 & 13 & \\
\hline \multirow{2}{*}{2} & $B$ & $70 \%$ & $\mathrm{NI}$ & $50 \%$ & NO LOH & $45 \%$ & NO LOH & $\begin{array}{ll}\mathrm{NI} \\
\end{array}$ & $74 \%$ & NO LOH & NO LOH & $60 \%$ & NO LOH & $81 \%$ & $\mathrm{NI}$ & $59 \%$ & 7 & 12 & \multirow{2}{*}{3} \\
\hline & $M$ & $85 \%$ & $\mathrm{NI}$ & NO LOH & NO LOH & NO LOH & NO LOH & $\mathrm{NI}$ & $98 \%$ & NO LOH & NO LOH & NO LOH & NO LOH & $93 \%$ & $\mathrm{NI}$ & NO LOH & 3 & 12 & \\
\hline \multirow{2}{*}{3} & B & $\mathrm{NI}$ & NO LOH & NO LOH & $\mathrm{NI}$ & NO LOH & NO LOH & NO LOH & $\mathrm{NI}$ & NO LOH & $\mathrm{NI}$ & $\mathrm{NI}$ & NO LOH & $73 \%$ & NO LOH & NO LOH & 1 & 10 & \multirow{2}{*}{0} \\
\hline & $M$ & $\mathrm{NI}$ & NO LOH & NO LOH & $\mathrm{NI}$ & NO LOH & NO LOH & NO LOH & $\mathrm{NI}$ & NOLOH & $\mathrm{NI}$ & $\mathrm{NI}$ & NO LOH & NO LOH & NO LOH & NO LOH & 0 & 10 & \\
\hline \multirow{2}{*}{4} & $B$ & $\mathrm{NI}$ & $66 \%$ & NO LOH & $54 \%$ & $\mathrm{NI}$ & $\mathrm{NI}$ & NO LOH & NO LOH & $\mathrm{NI}$ & $78 \%$ & $69 \%$ & $\mathrm{NI}$ & $\mathrm{NI}$ & $84 \%$ & $56 \%$ & 6 & 9 & \multirow{2}{*}{4} \\
\hline & M & $\mathrm{NI}$ & $88 \%$ & NO LOH & NO LOH & $\mathrm{NI}$ & $\mathrm{NI}$ & $58 \%$ & $62 \%$ & $\mathrm{NI}$ & $94 \%$ & NOLOH & $\mathrm{NI}$ & $\mathrm{NI}$ & $99 \%$ & $65 \%$ & 6 & 9 & \\
\hline \multirow{2}{*}{5} & $B$ & $55 \%$ & NO LOH & NO LOH & $46 \%$ & $54 \%$ & NO LOH & $\mathrm{NI}$ & $71 \%$ & NO LOH & NO LOH & NO LOH & NO LOH & $82 \%$ & NO LOH & $\mathrm{NI}$ & 5 & 13 & \multirow{2}{*}{4} \\
\hline & $M$ & $47 \%$ & NO LOH & NO LOH & NO LOH & $75 \%$ & NO LOH & $\mathrm{NI}$ & $86 \%$ & NO LOH & $57 \%$ & NO LOH & NO LOH & $95 \%$ & NO LOH & $\mathrm{NI}$ & 5 & 13 & \\
\hline \multirow{2}{*}{\multicolumn{2}{|c|}{6}} & NO LOH & 61 & NO LOH & NO LOH & $\mathrm{NI}$ & NO LOH & 73 & NO LOH & NO LOH & NO LOH & $\mathrm{NI}$ & NO LOH & 91 & NO LOH & NO LOH & 3 & 13 & \multirow{2}{*}{0} \\
\hline & & NO LOH & NO LOH & 77 & 44 & $\mathrm{NI}$ & NO LOH & NO LOH & NO LOH & 60 & NO LOH & $\mathrm{NI}$ & NO LOH & 57 & NO LOH & NO LOH & 4 & 13 & \\
\hline \multirow{2}{*}{\multicolumn{2}{|c|}{7}} & NO LOH & $\mathrm{NI}$ & NO LOH & $\mathrm{NI}$ & NO LOH & NO LOH & NO LOH & $\mathrm{NI}$ & NO LOH & NO LOH & NO LOH & $\mathrm{NI}$ & NO LOH & NO LOH & $\mathrm{NI}$ & 0 & 10 & \multirow{2}{*}{0} \\
\hline & & 59 & $\mathrm{NI}$ & NO LOH & $\mathrm{NI}$ & 67 & NO LOH & NO LOH & $\mathrm{NI}$ & 49 & NO LOH & 66 & $\mathrm{NI}$ & NO LOH & NO LOH & $\mathrm{NI}$ & 4 & 10 & \\
\hline \multirow{2}{*}{\multicolumn{2}{|c|}{8}} & $\mathrm{NI}$ & NO LOH & NO LOH & $\mathrm{NI}$ & NO LOH & NO LOH & $\mathrm{NI}$ & 80 & NO LOH & NO LOH & $\mathrm{NI}$ & NO LOH & $\mathrm{NI}$ & 56 & $\mathrm{NI}$ & 2 & 9 & \\
\hline & & $\mathrm{NI}$ & NO LOH & 48 & $\mathrm{NI}$ & NO LOH & NO LOH & $\mathrm{NI}$ & NO LOH & 60 & NO LOH & $\mathrm{NI}$ & NO LOH & $\mathrm{NI}$ & NOLOH & $\mathrm{NI}$ & 1 & 9 & \\
\hline & & NO LOH & NO LOH & $\mathrm{NI}$ & NO LOH & 75 & NO LOH & NO LOH & NO LOH & NO LOH & $\mathrm{NI}$ & NO LOH & NO LOH & 58 & NO LOH & NO LOH & 2 & 13 & \\
\hline 9 & & NO LOH & NO LOH & $\mathrm{NI}$ & NO LOH & NO LOH & NO LOH & NO LOH & NO LOH & NO LOH & $\mathrm{NI}$ & NO LOH & NO LOH & 69 & 50 & 58 & 3 & 13 & 0 \\
\hline 40 & & 59 & NO LOH & NO LOH & NO LOH & NO LOH & $\mathrm{NI}$ & 66 & NO LOH & $\mathrm{NI}$ & NO LOH & NO LOH & $\mathrm{NI}$ & NO LOH & $\mathrm{NI}$ & NO LOH & 2 & 11 & \\
\hline 10 & & NO LOH & NO LOH & 49 & NO LOH & NO LOH & $\mathrm{NI}$ & NO LOH & NO LOH & $\mathrm{NI}$ & 72 & NO LOH & $\mathrm{NI}$ & NO LOH & $\mathrm{NI}$ & NO LOH & 2 & 11 & 0 \\
\hline 11 & & $\mathrm{NI}$ & $\mathrm{NI}$ & NO LOH & $\mathrm{NI}$ & NO LOH & NO LOH & $\mathrm{NI}$ & NO LOH & NO LOH & NO LOH & NO LOH & 63 & $\mathrm{NI}$ & NO LOH & NO LOH & 1 & 10 & \\
\hline 11 & & $\mathrm{NI}$ & $\mathrm{NI}$ & 58 & $\mathrm{NI}$ & NO LOH & 66 & $\mathrm{NI}$ & 89 & NO LOH & NO LOH & NO LOH & 50 & $\mathrm{NI}$ & NO LOH & NO LOH & 4 & 10 & 0 \\
\hline 12 & & NO LOH & $\mathrm{NI}$ & $\mathrm{NI}$ & NO LOH & NO LOH & $\mathrm{NI}$ & NO LOH & $\mathrm{NI}$ & \begin{tabular}{|l|}
78 \\
\end{tabular} & NO LOH & \begin{tabular}{|l|}
$\mathrm{NI}$ \\
\end{tabular} & NO LOH & NO LOH & NO LOH & $\mathrm{NI}$ & 1 & 9 & \\
\hline & & 58 & $\mathrm{NI}$ & $\mathrm{NI}$ & 93 & 68 & $\mathrm{NI}$ & NO LOH & $\mathrm{NI}$ & NO LOH & \begin{tabular}{|l|}
60 \\
\end{tabular} & $\mathrm{NI}$ & NOLOH & 96 & NO LOH & $\mathrm{NI}$ & 6 & 9 & 0 \\
\hline 13 & & NO LOH & NO LOH & NO LOH & NOLOH & $\mathrm{NI}$ & NO LOH & 64 & NO LOH & $\mathrm{NI}$ & $\mathrm{NI}$ & NO LOH & NO LOH & NOLOH & $\mathrm{NI}$ & NO LOH & 1 & 11 & \\
\hline & & NO LOH & NO LOH & \begin{tabular}{|l|}
48 \\
\end{tabular} & NO LOH & $\mathrm{NI}$ & NO LOH & NO LOH & NO LOH & $\mathrm{NI}$ & $\mathrm{NI}$ & \begin{tabular}{|l|}
78 \\
\end{tabular} & 46 & NO LOH & $\mathrm{NI}$ & 55 & 3 & 11 & 0 \\
\hline 14 & & NO LOH & NO LOH & $\mathrm{NI}$ & $\mathrm{NI}$ & $\mathrm{NI}$ & NO LOH & NO LOH & 67 & NO LOH & NO LOH & NOLOH & $\mathrm{NI}$ & $\mathrm{NI}$ & NO LOH & NO LOH & 1 & 10 & \\
\hline 14 & & NO LOH & NO LOH & $\mathrm{NI}$ & $\mathrm{NI}$ & $\mathrm{NI}$ & 55 & NO LOH & NO LOH & NO LOH & NO LOH & 66 & $\mathrm{NI}$ & $\mathrm{NI}$ & 49 & 78 & 4 & 10 & 0 \\
\hline 15 & & $\mathrm{NI}$ & 59 & NO LOH & NO LOH & NO LOH & $\mathrm{NI}$ & NO LOH & NO LOH & \begin{tabular}{|c|}
$\mathrm{NI}$ \\
\end{tabular} & $\mathrm{NI}$ & NO LOH & NO LOH & 88 & $\mathrm{NI}$ & $\mathrm{NI}$ & 2 & 9 & 0 \\
\hline & & $\mathrm{NI}$ & NO LOH & NO LOH & \begin{tabular}{|l|}
59 \\
\end{tabular} & NO LOH & $\mathrm{NI}$ & NO LOH & 62 & $\mathrm{NI}$ & $\mathrm{NI}$ & 49 & NO LOH & 56 & $\mathrm{NI}$ & $\mathrm{NI}$ & 4 & 9 & 0 \\
\hline 16 & & NO LOH & 55 & $\mathrm{NI}$ & $\mathrm{NI}$ & NO LOH & 67 & NO LOH & NO LOH & 71 & $\mathrm{NI}$ & NO LOH & NO LOH & 93 & $\mathrm{NI}$ & $\mathrm{NI}$ & 4 & 10 & \\
\hline & & NO LOH & NO LOH & $\mathrm{NI}$ & $\mathrm{NI}$ & 52 & NO LOH & NO LOH & 60 & NO LOH & $\mathrm{NI}$ & NO LOH & NO LOH & 78 & $\mathrm{NI}$ & $\mathrm{NI}$ & 3 & 10 & 0 \\
\hline 17 & & NO LOH & 58 & NO LOH & NO LOH & NO LOH & NO LOH & NO LOH & NO LOH & NO LOH & NO LOH & NO LOH & NO LOH & $\mathrm{NI}$ & NO LOH & NO LOH & 1 & 14 & \\
\hline 18 & & NO LOH & NO LOH & NO LOH & NO LOH & NO LOH & NO LOH & NO LOH & 68 & NO LOH & NO LOH & NO LOH & NO LOH & $\mathrm{NI}$ & NO LOH & NO LOH & 1 & 14 & 0 \\
\hline
\end{tabular}

Figure 3 Molecular analysis results of five patients with unilateral breast cancer and axillary lymph metastases (patients 1-5) and 12 patients with synchronous bilateral breast carcinoma (patients 6-17).

cancer formation since it is not likely for a single cancer to lose a phenotypically enhancing mutation and reacquire the same mutation on the opposite allele.

Fractional allelic loss, a measure to cumulative mutational load, varied widely across the different patients ranging from as low as $0 / 10$ (patient 3 ) to as high as $6 / 9$ (patient 12). The greater the number of mutations present for comparison, the easier the distinction could be made with respect to cancer relatedness. However, even in those patients with few mutations, independent primary cancer formation was supported based on the fact that metastatic spread, in general required a high FAL index. It has been our experience that low-grade neoplasms tend to lack high levels of acquired mutations.

\section{Discussion}

Bilateral breast carcinoma represents a relatively small proportion of all breast carcinoma cases in the United States each year. However, the incidence of bilateral breast carcinoma is rising as a direct result of improved detection capabilities. ${ }^{28}$ Only a few reports have investigated the genetic findings in synchronous bilateral breast cancer. ${ }^{19-22,25}$
Whether bilateral breast cancers have an independent origin or are the result of a breast-to-breast metastasis may be crucial since the therapeutic management of independent breast carcinoma is different from the treatment of metastatic disease. ${ }^{12,16,19,29}$ Dissimilarity in histology between bilateral breast carcinoma in the same patient was often cited in support of the independent primaries theory. In contrast, positive correlations in histologic subtype, tumor grade, and hormonal receptor status between the two tumors have been considered to be indicative of a single-cell origin with secondary metastatic spread of cancer cells to the opposite breast. However, histologic features, such as histologic differentiation, type, grading, and presence of in situ component, appear to be of little discriminatory value to differentiate metastatic vs independent two primaries. Hungness et $a l^{23}$ showed that there was a similar histologic type of bilateral breast carcinoma in most of their patients. Coradini et $a{ }^{29}$ reported that ER and PgR levels in the primary and contralateral breast tumors did not differ significantly. In our study, similar histologic type and nuclear grade were seen in both bilateral tumors in $58 \%$, ER positivity in $75 \%$, and HER-2 status in $67 \%$. Pandis et $a l^{19}$ concluded that in situ lesions could no longer considered as a 
criterion for de novo carcinogenesis. Based on the genetic profile, Imyanitov et $a l^{20}$ found two independent primaries despite their similar histologic differentiation and nodal metastases at the time of diagnosis. Similarly, two of our cases showed lymph node metastases and proved to be independent primaries. In our study, the histologic parameters allowed us to diagnosis de novo synchronous bilateral breast cancer in 5/12 (42\%) cases. This would signify an important reevaluation of the histological criteria commonly used to differentiate between primary vs metastatic lesions. In addition, differences in the hormone receptor status have no discriminatory value since differences between the primary breast cancer and its metastases have been reported. ${ }^{29,30,31}$

Although some studies showed that synchronous carcinoma behaves more aggressively than metachronous carcinomas, ${ }^{8}$ other investigators have concluded that patients with synchronous bilateral breast carcinoma have the same or a favorable prognosis as their unilateral counterpart breast carcinoma when adjusted for maximum stage. ${ }^{6,21,29,30,31-35}$ Similar to Intra et $a l^{30}{ }^{30} 11 / 24$ $(46 \%)$ of our patients were classified as grade 2 , while high-grade tumors (grade 3) were infrequent in synchronous bilateral carcinoma. ${ }^{36}$ Moreover, patients with synchronous bilateral carcinoma have a higher rate of ER and PgR positivity than that in patients with unilateral breast cancer, suggesting less aggressive biologic behavior. ${ }^{37}$ In our study, synchronous bilateral breast cancer patients showed favorable characteristics, such as low histologic grade, absence of lymphovascular invasion, lymph node metastases, and ER and PgR positivity in 87.5 and $71 \%$, respectively. Although most of the tumors in our study were infiltrating ductal carcinoma, our studied population shows a higher proportion of special tumor types, such as pure lobular, tubular, and mucinous $(5 / 24,21 \%)$. Safal et $a l^{38}$ reported a high percentage of HER-2 overexpression in $71 \%$ of their cases. On the other hand, similar to our results, Matsuo et $a l^{37}$ reported a HER-2 positivity rate in $18 \%$ of their cases ( $25 \%$ in our study).

It has been suggested that synchronous bilateral breast cancer is associated with a 39\% incidence of positive family history and associated with young age, ${ }^{6,10}$ as compared with a $5-10 \%$ incidence generally reported for breast carcinoma patients. ${ }^{39}$ Among BRCA1 carrier with a first breast carcinoma, the risk for a contralateral cancer is estimated at $48 \%$ by age 50 and $64 \%$ by age $70 .{ }^{40}$ Others reported lower median age around 50 years. ${ }^{30,41-43}$ However, we did not find a trend toward young age in our patients (median age, 74 years), consistent with other studies. ${ }^{12,16,18,23,30,38}$ The percentage of postmenopausal women was variable ranging from $29 \%{ }^{42}$ to $62 \% .^{12}$ In our study, majority of the patients with synchronous bilateral breast carcinoma were postmenopausal $(11 / 12,92 \%)$. The clinical history of our patients provided no evidence to suggest that our series might include cases of familial breast cancer, and the high mean age of our patients supports this assumption. Bilaterality does not necessarily reflect a genetic predisposition, ${ }^{44}$ unless it is associated with an early age of onset ${ }^{45}$

Tumor allelotyping is considered a powerful method for examining the clonal origin of multiple malignancies. ${ }^{46-48}$ Allelotyping provides a single tumor-specific 'cytogenetic fingerprint' with a high, but limited, number of molecular markers investigated. It is accepted that multiple neoplasms presenting different allotypes possess distinct clonal origins, whereas concordant allelic profiles suggest a monoclonal origin of the tumors. ${ }^{4-49}$ In the present study, the allelotyping data provided clear proof of an independent clonal origin of all our cases of synchronous bilateral breast carcinomas. In contrast, our cases with LN metastases showed identical genotyping data, allowed us to determine metastatic disease. ${ }^{20}$

Available genotypic data have shown that invasive carcinomas and their synchronous metastases usually exhibit the same genetic profile. ${ }^{50,51}$ In our study, a detailed mutational profile encompassing 15-20 molecular markers is generated from multiple representative tissue sites within a given neoplastic deposit. The use of multiple targets helps described intratumoral heterogeneity. ${ }^{52}$ Given that carcinogenesis is a multistep process of irreversible mutation acquisition causally associated with enhanced neoplastic phenotype, mutations acquired early are expected to be clonally expanded in metastasis derived from that cancer. Therefore, the initial molecular lesions detected in the primary tumor will be retained in the corresponding asynchronous distant metastases, with a noticeable accumulation of additional genetic events. ${ }^{51}$ In each case, these discordant mutations will appear as later temporal events and will, therefore, not obscure the relatedness between the two tumors. In the case of independent primary cancer formation, it is statistically unlikely that two primary cancers will accumulate the identical constellation, temporal profile, and specific allele patterns of mutational change. $^{46}$ However, there may be a very small chance that $\mathrm{LOH}$ of the same allele in two different specimens results from two independent events that coincidentally give the same result. ${ }^{12}$ This theory may explain the few cases of synchronous bilateral breast carcinomas in literature that common clonality could not be entirely excluded. ${ }^{11,19-21,29}$

There is debate regarding the surgical management of patients with synchronous bilateral breast carcinoma. Similar to our experience, most studies have shown high incidence of bilateral mastectomies performed in their patients. ${ }^{23}$ This aggressive approach was adopted based on the assumption that synchronous bilateral breast carcinoma has a worse prognosis than unilateral breast cancer. Recent studies showed that bilateral breast conservation is a reasonable approach without affecting overall 
and disease-free survival, while enhancing patient cosmesis. ${ }^{30,33,34}$

In conclusion, this study supports the observations concerning the independent clonal origin of most, if not all, synchronous bilateral breast carcinomas. These findings appear to have practical impact, as clinical management of localized breast cancer is critically different from treatment of metastatic disease.

\section{Acknowledgement}

This work was accepted as a poster presentation, in part, at the 2006 annual meeting of the United States-Canadian Academy of Pathology, Atlanta, GA, USA.

\section{References}

1 Pomerantz RA, Murad T, Hines JR. Bilareal breast cancer. Am Surg 1989;55:441-444.

2 Kurtz JM, Amalric R, Brandone H, et al. Contralateral breast cancer and other second malignancies in patients treated by breast-conserving therapy with radiation. Int J Radiat Oncol Biol Phys 1988;15: 277-284.

3 Harvey EB, Brinton LA. Second cancer following cancer of the breast in Connecticut, 1935-82. Natl Cancer Inst Monogr 1985;68:99-112.

4 Donovan AJ. Bilateral breast cancer. Surg Clin North Am 1990;70:1141-1149.

5 Chaudary MA, Millis RR, Hoskins EO, et al. Bilateral primary breast cancer: a prospective study of disease incidence. Br J Surg 1984;71:711-714.

6 de la Rochefordiere A, Asselain B, Scholl S, et al. Simultaneous bilateral breast carcinomas: a retrospective review of 149 cases. Int J Radiat Oncol Biol Phys 1994;30:35-41.

7 Jobsen JJ, van der Palen J, Ong F, et al. Synchronous, bilateral breast cancer: prognostic value and incidence. Breast 2003;12:83-88.

8 Kollias J, Ellis IO, Elston CW, et al. Prognostic significance of synchronous and metachronous bilateral breast cancer. World J Surg 2001;25:1117-1124.

9 Fisher ER, Fisher B, Sass R, et al., collaborating NSABP investigators. Pathologic findings from the National Surgical Adjuvant Breast Project (protocol No. 4). Cancer 1984;54:3002-3011.

10 Kelmendi de Ustaran J, Meiss RP. Primary synchronous bilateral breast cancer: epidemiological approach. Breast Cancer Res Treat 1988;12:311-314.

11 Chunder N, Roy A, Roychoudhury S, et al. Molecular study of clonality in multifocal and bilateral tumors. Pathol Res Pract 2004;200:735-741.

12 Burns PE, Dabbs K, May C, et al. Bilateral breast cancer in northern Alberta: risk factors and survival patterns. Can Med Assoc J 1984;130:881-886.

13 Rosselli Del Turco M, Ciatto S, Perigli G, et al. Bilateral cancer of the breast. Tumori 1982;68:155-160.

14 Mueller CB, Ames F. Bilateral carcinoma of the breast: frequency and mortrality. Can J Surg 1978;21:459-465.

15 Imyanitov EN, Hanson KP. Molecular pathogenesis of bilateral breast cancer. Cancer Lett 2003;191:1-7.
16 Dawson PJ, Maloney T, Gimotty P, et al. Bilateral breast cancer; one disease or two? Breast Cancer Res Treat 1991;19:233-244.

17 Sterns EE, Fletcher WA. Bilateral cancer of the breast: a review of clinical, histologic, and immunohistologic characteristics. Surgery 1991;110:617-622.

18 Kiang DT, Kennedy BJ, Snover DC. Biological and histological characteristics of simultaneous bilateral breast cancer. Lancet 1980;2:1105-1107.

19 Pandis N, Teixeira MR, Gerdes AM, et al. Chromosome abnormalities in bilateral breast carcinomas. Cytogenetic evaluation of the clonal origin of multiple primary tumors. Cancer 1995;76:250-258.

20 Imyanitov EN, Suspitsin EN, Grigoriev MY, et al. Concordance of allelic imbalance profiles in synchronous and metachronous bilateral breast carcinomas. Int J Cancer 2002;100:557-564.

21 Shibata A, Tsai YC, Press MF, et al. Clonal analysis of bilateral breast cancer. Clin Cancer Res 1996;2: 743-748.

22 Teixeira MR, Ribeiro FR, Torres L, et al. Assessment of clonal relationships in ipsilateral and bilateral multiple breast carcinomas by comparative genomic hybridization and hierarchical clustering analysis. $\mathrm{Br} \mathrm{J}$ Cancer 2004;91:775-782.

23 Hungness ES, Safa M, Shaughnessy EA, et al. Bilateral synchronous breast cancer: mode of detection and comparison of histologic features between the 2 breasts. Surgery 2000;128:702-707.

24 Kollias J, Man S, Marafie M, et al. Loss of heterozygosity in bilateral breast cancer. Breast Cancer Res Treat 2000;64:241-251.

25 Tse GM, Kung FY, Chan AB, et al. Clonal analysis of bilateral mammary carcinomas by clinical evaluation and partial allelotyping. Am J Clin Pathol 2003;120: 168-174.

26 Hall NJ, Evans AJ, Kollias J, et al. Bilateral breast carcinomas: do they have similar mammographic features? Clin Radiol 1999;54:434-437.

27 Murphy TJ, Conant EF, Hanau CA, et al. Bilateral breast carcinoma: mammographic and histologic correlation. Radiology 1995;195:617-621.

28 Hislop TG, Elwood JM, Coldman AJ, et al. Second primary cancers of the breast: incidence and risk factors. Br J Cancer 1984;49:79-85.

29 Coradini D, Oriana S, Mariani L, et al. Is steroid receptor profile in contralateral breast cancer a marker of independence of the corresponding primary tumor? Euro J Cancer 1998;34:825-830.

30 Intra M, Rotmensz N, Viale G, et al. Clinicopathologic characteristics of 143 patients with synchronous bilateral invasive breast carcinomas treated in a single institution. Cancer 2004;101:905-912.

31 Janschek E, Kandioler-Eckersberger D, Ludwig C, et al. Contralateral breast cancer: molecular differentiation between metastasis and second primary cancer. Breast Cancer Res Treat 2001;67:1-8.

32 Heron DE, Komarnicky LT, Hyslop T, et al. Bilateral breast carcinoma: risk factors and outcomes for patients with synchronous and metachronous disease. Cancer 2000;88:2739-2750.

33 Lee MM, Chen LM, Heimann R, et al. Efficacy of breast conservation therapy in early stage bilateral breast cancer. Proc Int J Radiat Oncol Biol Phys 1996;36:281.

34 Gollamudi SV, Gelman RS, Schneider LJ, et al. Breastconserving therapy for stage I-II synchronous bilateral breast carcinoma. Cancer 1997;79:1362-1369. 
35 Mose S, Adamietz IA, Thilmann C, et al. Bilateral breast carcinoma versus unilateral disease. Review of 498 patients. Am J Clin Oncol 1997;20:541-545.

36 Tsuda H, Takarabe T, Akashi-Tanaka S, et al. Evaluation of histopathological criteria for identifying nodenegative breast cancer with high risk of early recurrence in the NSAS-BC protocol study. Breast Cancer 2000;7:201-209.

37 Matsuo K, Fukutomi T, Akashi-Tanaka S, et al. Histological grade, p53, HER2 and hormone receptor status of synchronous bilateral breast carcinoma. Breast Cancer 2002;9:127-133.

38 Safal M, Lower EE, Hasselgren PO, et al. Bilateral synchronous breast cancer and HER-2/neu overexpression. Breast Cancer Res Treat 2002;72:195-201.

39 Tulusan AH, Ronay G, Egger H, et al. A contribution to the natural history of breast cancer. V. Bilateral primary breast cancer: incidence, risks and diagnosis of simultaneous primary cancer in the opposite breast. Arch Gynecol 1985;237:85-91.

40 Kinne DW. Special therapeutic problems: management of the contralateral breast. In: Harris JR, Hellman S, Henderson IC, Kinne DW (eds). Breast Diseases. JB Lippincott: Philadelphia, 1991, pp 827-833.

41 Green MH. Genetic of breast cancer. Mayo Clin Proc 1997;72:54-65.

42 Gollamudi SV, Gelman RS, Peiro G, et al. Breastconserving therapy for stage I-II synchronius bilateral breast carcinoma. Cancer 1997;79:1362-1369.

43 Newman LA, Sahin AA, Cunningham JE, et al. A casecontrol study of unilateral and bilateral breast carcinoma patients. Cancer 2001;91:1845-1853.
44 Steinmann D, Bremer M, Rades D, et al. Mutations of the BRCA1 and BRCA2 genes in patients with bilateral breast cancer. Br J Cancer 2001;85:850-858.

45 Bremer M, Steinmann D, Dork T, et al. Bilateral breast carcinoma and local recurrence: prevalence of BRCA-1 and BRCA-2 gene mutations in an unselected patient sample. Strehlenther Onkol 2001; 177:325-329.

46 Gershoni-Baruch R, Dagan E, Fried G, et al. BRCA1 and BRCA2 founder mutations in patients with bilateral breast cancer. Eur J Hum Genet 1999;7:833-836.

47 Fujii H, Marsh C, Cairns P, et al. Genetic divergence in the clonal evolution of breast cancer. Cancer Res 1996; 56:1493-1497.

48 Agelopoulos K, Tidow N, Korsching E, et al. Molecular cytogenetic investigations of synchronous bilateral breast cancer. J Clin Pathol 2003;56:660-665.

49 Scholes AG, Woolgar JA, Boyle MA, et al. Synchronous oral carcinomas: independent or common clonal origin? Cancer Res 1998;58:2003-2006.

50 Takahashi T, Habuchi T, Kakehi Y, et al. Clonal and chronological genetic analysis of multifocal cancers of the bladder and upper urinary tract. Cancer Res 1998;58:5835-5841.

51 Bonsing BA, Devilee P, Cleton-Jansen AM, et al. Evidence of limited molecular genetic heterogeneity as defined by allelotyping and clonal analysis in nine metastatic breast carcinomas. Cancer Res 1993;53: 3804-3811.

52 Lichy JH, Dalbegue F, Zavar M, et al. Genetic heterogeneity in ductal carcinoma of the breast. Lab Invest 2000;80:291-301. 